\title{
Effects of lifestyle and single nucleotide polymorphisms on breast cancer risk: a case-control study in Japanese women
}

\author{
Taeko Mizoo ', Naruto Taira" ${ }^{*}$, Keiko Nishiyama', Tomohiro Nogami', Takayuki Iwamoto', Takayuki Motoki', \\ Tadahiko Shien', Junji Matsuoka', Hiroyoshi Doihara', Setsuko Ishihara², Hiroshi Kawai ${ }^{3}$, Kensuke Kawasaki ${ }^{4}$, \\ Youichi Ishibe ${ }^{5}$, Yutaka Ogasawara ${ }^{6}$, Yoshifumi Komoike ${ }^{7}$ and Shinichiro Miyoshi ${ }^{1}$
}

\begin{abstract}
Background: Lifestyle factors, including food and nutrition, physical activity, body composition and reproductive factors, and single nucleotide polymorphisms (SNPs) are associated with breast cancer risk, but few studies of these factors have been performed in the Japanese population. Thus, the goals of this study were to validate the association between reported SNPs and breast cancer risk in the Japanese population and to evaluate the effects of SNP genotypes and lifestyle factors on breast cancer risk.
\end{abstract}

Methods: A case-control study in 472 patients and 464 controls was conducted from December 2010 to November 2011. Lifestyle was examined using a self-administered questionnaire. We analyzed 16 breast cancer-associated SNPs based on previous GWAS or candidate-gene association studies. Age or multivariate-adjusted odds ratios (OR) and 95\% confidence intervals $(95 \% \mathrm{Cl})$ were estimated from logistic regression analyses.

Results: High BMl and current or former smoking were significantly associated with an increased breast cancer risk, while intake of meat, mushrooms, yellow and green vegetables, coffee, and green tea, current leisure-time exercise, and education were significantly associated with a decreased risk. Three SNPs were significantly associated with a breast cancer risk in multivariate analysis: rs2046210 (per allele $\mathrm{OR}=1.37$ [95\% Cl: 1.11-1.70]), rs3757318 (OR=1.33[1.05-1.69]), and rs3803662 (OR = 1.28 [1.07-1.55]). In 2046210 risk allele carriers, leisure-time exercise was associated with a significantly decreased risk for breast cancer, whereas current smoking and high BMI were associated with a significantly decreased risk in non-risk allele carriers.

Conclusion: In Japanese women, rs2046210 and 3757318 located near the ESR1 gene are associated with a risk of breast cancer, as in other Asian women. However, our findings suggest that exercise can decrease this risk in allele carriers.

Keywords: Japanese women, Asian, Breast cancer, Lifestyle, Leisure-time exercise, Parity, Single nucleotide polymorphisms, rs2046210, rs3757318, ESR1

\section{Background}

Data in the National Statistics of Cancer Registries by Region (1975-2004) indicate that the prevalence of breast cancer in Japan has increased steadily since 1975. More than 60,000 patients had breast cancer in 2008 and the mammary gland is the most common site of a

\footnotetext{
*Correspondence: ntaira@md.okayama-u.ac.jp

'Department of General Thoracic Surgery and Breast and Endocrinological Surgery, Okayama University Graduate School of Medicine, Dentistry, and Pharmaceutical Sciences, 2-5-1 Shikata-cho, Okayama-city, Okayama 700-8558, Japan

Full list of author information is available at the end of the article
}

malignant tumor in Japanese women [1]. Additionally, the Vital Statistics Japan database of the Ministry of Health, Labor and Welfare indicates that mortality due to breast cancer in Japan has increased since 1960, with more than 10,000 deaths from breast cancer in 2011 [2].

The relationship of lifestyle factors, including food and nutrition, physical activity, body composition, environmental factors, and reproductive factors, with breast cancer risk have been widely studied, mainly in Europe and the United States, and much evidence linking cancer to these factors has been accumulated. According to the

\section{Biomed Central}


2007 World Cancer Research Fund/American Institute for Cancer Research (WCRF/AICR) Second Expert Report, the evidence that breastfeeding decreases the breast cancer risk and that alcohol increases this risk is described as "convincing" [3]. In postmenopausal women, evidence that body fat and adult attained height increase breast cancer risk is also stated to be "convincing". However, the evidence of a relationship of other foods with breast cancer risk remains at the level of "limited-no conclusion". Thus, it is important to identify risk factors for breast cancer with the goal of prevention through efficient screening and surveillance.

In the United States, a breast cancer risk assessment tool based on a statistical model known as the "Gail model" has been produced by the National Cancer Institute $(\mathrm{NCI})[4,5]$. However, this model has been developed from epidemiological data in Caucasians and it may be inappropriate to apply the Gail model in the Japanese population [6]. However, there are few epidemiological studies of breast cancer risk in Japanese women and a breast cancer risk model applicable to Japanese women has yet to be established.

Regarding genetic factors, genome-wide association studies (GWAS) have identified several breast cancer susceptibility single nucleotide polymorphisms (SNPs) [7]. However, most of these studies were also conducted in subjects with European ancestry, with some in populations with Chinese ancestry or in African Americans. There is only one such study in subjects with Japanese ancestry. However, allele frequencies related to breast cancer risk and the extent of linkage disequilibrium differ among races. Thus, the validity of the reported associations of SNPs with breast cancer needs to be tested in a Japanese population.

Current findings suggest that the interactions between breast cancer susceptibility SNPs and breast cancer risk are not as strong as those for BRCA1 or BRCA2 gene mutation. However, carriers of risk SNP alleles are more common compared with carriers of BRCA1 or BRCA2 mutation. Evaluation of the need to incorporate SNPs into a breast cancer risk model requires examination of the influence of these SNPs and established breast cancer risk factors to determine whether these are mutually confounding factors. Moreover, such findings might allow risk allele carriers to reduce their incidence of breast cancer through guidance on lifestyle habits.

The current study was performed to add to the relatively small number of studies that have examined genomic factors such as SNPs in combination with non-genomic factors such as those associated with lifestyle. We first aimed to validate whether reported breast cancer susceptibility SNPs are applicable in the Japanese population. We then examined the possible confounding effects on breast cancer risk of SNPs and lifestyle factors such as food, nutrition, physical activity, body composition, environment factors and reproductive factors.

\section{Methods \\ Subjects}

A multicenter population-based case-control study was conducted between December 2010 and November 2011 in Japan. The subjects were consecutive patients with noninvasive or invasive breast cancer aged over 20 years old who were treated at Okayama University Hospital, Okayama Rousai Hospital and Mizushima Kyodo Hospital in Okayama and at Kagawa Prefecture Central Hospital in Kagawa. The controls were women aged over 20 years old without a history of breast cancer who underwent breast cancer screening at Mizushima Kyodo Hospital and Okayama Saiseikai Hospital in Okayama and at Kagawa Prefectural Cancer Detection Center in Kagawa. All subjects gave written informed consent before enrollment in the study. A blood sample $(5 \mathrm{ml})$ used for SNP analysis was collected from each subject. Subjects were also given questionnaires that they completed at home and mailed back to Okayama University Hospital. The study was approved by the institutional ethics committee on human research at Okayama University.

\section{Survey of lifestyle}

A survey of lifestyle was performed using an 11-page self-administered questionnaire that included questions on age, height and body weight (current and at 18 years old), cigarette smoking, alcohol drinking, intake of 15 foods items, intake of 4 beverages, leisure-time exercise (current and at 18 years old), menstruation status, age at first menstruation, age at first birth, parity, breastfeeding, age at menopause, hormone replacement therapy (HRT), history of benign breast disease, familial history of breast cancer, and education. Controls answered the survey based on their current status and patients referred to their prediagnostic lifestyle.

Body mass index (BMI) was calculated as body weight/ square of height. Former or current alcohol drinkers were asked to give the frequency per week and type of drink usually consumed (beer, wine, sake, whisky, shochu, or others). The alcoholic content of each drink was taken to be $8.8 \mathrm{~g}$ per glass $(200 \mathrm{ml})$ of beer, and $20 \mathrm{~g}$ per glass of sake $(180 \mathrm{ml})$, wine $(180 \mathrm{ml})$, shochu $(110 \mathrm{ml})$ and whisky $(60 \mathrm{ml})$ [8]. Alcohol intake per day (g/day) was calculated as follows: (total alcohol content per occasion $\times$ frequency of consumption per week)/7. Women who currently engaged in leisure-time exercise were asked to give the intensity of physical activity per occurrence and frequency per week. Metabolic equivalent (MET) values of 10, 7, 4, and 3 METs were assigned for strenuous-, moderate-, low-, and very low intensity activities per occurrence, respectively [9], to allow calculation of the intensity of 
physical activity in leisure-time exercise per week (METs/ week). A family history of breast cancer included mother, sisters and daughters (first-degree family history). History of benign breast disease included the non-cancerous breast. Clinical data on patients were obtained from hospital medical records.

\section{Selection of SNPs}

Sixteen breast cancer-associated SNPs were identified from previous GWAS [7] and candidate-gene association studies: ATM/11q22-rs1800054 [10], 8q24-rs1562430 [11], MAP3K1/Chr5-rs889132 [10,12], 2q-rs4666451 [10], 8q24-rs13281615 [10,12,13], TTNT3/11p15-rs909116 [11], 5q-rs30099 [10], IGF1/12q23.2-795399 [10,14], ESR1/6q25.1-rs2046210 [15,16], CAPSP8/2q33-34-rs1045485 [10], 2q35-rs13387042 [10], ESR1/6q25.1-rs3757318 [11], TNRC9/16q12-rs3803662 [12,17], FGFR2/10q26rs2981282 [10,12], LSP1/11p15.5-rs381798 [12], and HCN1/5p12- rs98178 [10]. Risk alleles associated with breast cancer were identified with reference to the Japanese Single Nucleotide Polymorphism (JSNP) database [18].

\section{SNP genotyping}

Genomic DNA was isolated from whole blood with a TaqMan $^{\oplus}$ Sample-to-SNP ${ }^{\mathrm{rm}}$ kit (Applied Biosystems, Foster City, CA, USA). Samples were analyzed by a TaqMan genotyping assay using the StepOne ${ }^{\mathrm{TM}}$ real-time polymerase chain reaction (PCR) system (Applied Biosystems) in a 96-well array plate that included four blank wells as negative controls. The PCR profile consisted of an initial denaturation step at $95^{\circ} \mathrm{C}$ for $10 \mathrm{~min}, 40$ cycles of $92^{\circ} \mathrm{C}$ for $15 \mathrm{sec}$, and $60^{\circ} \mathrm{C}$ for $1 \mathrm{~min}$. PCR products were analyzed by StepOne ${ }^{\mathrm{Ts}}$ Software Ver2.01 (Applied Biosystems). To assess the quality of genotyping, we conducted re-genotyping of a randomly selected $5 \%$ of samples and obtained $100 \%$ agreement.

\section{Statistical analysis}

For all analyses, significance was defined as a p-value $<0.05$. Associations between lifestyle and breast cancer risk were estimated by computing age adjusted odds ratios (OR) and their 95\% confidence intervals (CI) from logistic regression analyses. Height was categorized as $\leq 150$, 151$155,156-160$ and $>160$ according to quartile. Weight was categorized as <50, 50-54.9, 55-59.9 and $\geq 60$ according to quartile. BMI was categorized as $\leq 20,20-21.9,22-23$ and $\geq 24$ according to quartile. Alcohol intake per day (g/day) was categorized as $0,<5,5-10$ and $\geq 10 \mathrm{~g} /$ day according to quartile. Food intake, including meat, fish, egg, soy, milk, fruits, green and yellow vegetables and mushrooms, was categorized as $\leq 1,2-4$ and 5 times/week. Beverage intake including coffee and green tea was categorized as $\leq 1,2-3$ and $\geq 3$ cups/day. Intensity of physical activity in leisure time was categorized as $0,<6,6-11.9,12-23.9$ and $\geq 24$ METs/week. Age at menarche was classified as $\leq 12,13$ and $\geq 14$ years old, parity as $0,1-2$ and $\geq 3$, and age at first childbirth as $<25,25-29$ and $\geq 30$ years old. Education level was categorized as high school or less, two-year college, and university or higher.

In analysis of SNPs, accordance with the HardyWeinberg equilibrium was checked in controls using a chi-squared test. The associations between genotype and the risk of breast cancer were estimated by computing OR and the $95 \%$ CI from logistic regression analyses. Per allele OR was calculated using 0,1 or 2 copies of the risk allele (a) as a continuous variable. The reported OR and 95\% CI denote the risk difference when increasing the number of risk alleles by one. Two models of analyses were performed, with the first model adjusted only for age and the second model adjusted for factors that were significantly associated with breast cancer risk in this study (multivariate adjustment).

For SNPs associated with breast cancer, we classified subjects as risk allele carriers or non-risk allele carriers and examined associations of lifestyle factors with breast cancer risk in these subgroups. Two models were also used in this analysis, with the second model adjusted for factors that were significantly associated with breast cancer risk in the first model.

All statistical analyses were performed with Statistical Analysis System software JMP version 9.0.3 (SAS Institute).

\section{Results}

A total of 515 patients and 527 controls agreed to participate in the study and gave written informed consent. Of these women, 476 patients (92.4\%) and 464 controls (88.8\%) returned self-administered questionnaires. In 2 cases, blood samples could not be obtained because of brittle vessels and in another 2 cases SNP genotyping could not be performed because of poor DNA amplification. Thus, the final data set for analysis included 472 patients and 464 controls with completed questionnaires and SNP genotyping.

Adjusted OR with 95\% CIs for lifestyle factors are shown in Table 1 . BMI $\geq 24$ (vs. 20-21.9) and current or former smoker (vs. never) were associated with a significantly increased risk for breast cancer. Meat intake $\geq 2$ times/week (vs. $\leq$ once/week), mushroom intake (vs. $\leq$ once/ week), yellow and green vegetable intake (vs. $\leq$ once/week), coffee intake 2-3 cups/day (vs. <1 cup/day), green tea intake 2-3 cups/day (vs. <1 cup/day), current leisure-time exercise (vs. none), intensity of physical activity in leisuretime exercise 6-23.9 METS/week (vs. 0 METS/week), and university education (vs. high school or less) were all associated with a significantly decreased risk for breast cancer. Height, alcohol intake, age at first menstruation, parity, age at first birth, and familial history of breast cancer have generally been considered to be associated with breast 
cancer risk, but did not show a significant association in this study.

In analysis of SNPs, deviation from the Hardy-Weinberg equilibrium ( $\mathrm{P}<0.05$ by chi square test) was found for rs1800054 and rs1045485, and thus these SNPs were excluded from analysis. The minor allele frequencies were $<0.05$ for rs4666451 and rs104548, and these SNPs were also excluded, leaving 12 SNPs for analysis. Multivariate ORs were adjusted for factors that were found to be significantly associated with breast cancer: BMI, smoking status, meat intake, mushroom intake, yellow and green vegetable intake, coffee intake, green tea intake, leisuretime exercise and education level.

Age adjusted ORs and multivariate ORs with 95\% CIs for independent SNPs in all subjects and in subjects stratified by menopausal status are shown in Table 2. In all women, three SNPs were significantly associated with breast cancer risk in multivariate adjustment: rs2046210 (per allele OR $=1.37$ [95\% CI:1.11-1.70]), rs3757318 (per allele $\mathrm{OR}=1.33$ [1.05-1.69] and rs3803662 (per allele = 1.28 [1.07-1.55]). rs2046210 and rs3757318, both of which are located on 6q25.1, are not in strong linkage disequilibrium $(\mathrm{LD})(\mathrm{D}=0.68, \mathrm{r} 2=0.21)$ according to Hap-Map JTP [19]. Among pre-menopausal women, s3803662 (per allele $\mathrm{OR}=1.58$ [95\% CI: 1.17-2.16]) and rs2046210 (per allele $\mathrm{OR}=1.70$ [95\% CI: 1.24-2.35]) were significantly associated with breast cancer risk in multivariate adjustment. Among post-menopausal women, there were no SNPs significantly associated with breast cancer risk.

A subgroup analysis was performed for rs2046210 and rs3757318. For rs2046210, leisure time exercise was associated with a significantly decreased breast cancer risk in risk allele carriers $(\mathrm{AA}+\mathrm{AG})$, but not in non-risk allele carriers (GG). In contrast, $\mathrm{BMI} \geq 24$ and current smoking were associated with a significantly increased breast cancer in non-risk allele carriers (GG), but not in risk allele carriers (AA + AG). Intensity of physical activity in leisure exercise of 12.0-23.9 METS/week and university education were associated with breast cancer risk in risk allele and non-risk allele carriers (Table 3). For rs3757318, BMI $\geq 24$ was associated with a significantly increased breast cancer risk in risk allele carriers (GG), but not in risk allele carriers $(\mathrm{AA}+\mathrm{AG})$. University education and current smoking were associated with breast cancer risk in risk allele and non-risk allele carriers (Table 4).

\section{Discussion}

Associations of breast cancer risk with lifestyle factors and SNPs alone and in combination were examined in a casecontrol study in 472 patients and 464 controls. Reproductive factors such as early age at first menstruation, late age at menopause, late age at first birth, nulliparity, and no breastfeeding have been associated with an increase in breast cancer risk [20], including in the Japanese population
[21]. In our study, parity and breastfeeding showed a tendency for an association with decreased breast cancer risk, but this association was not significant; and age at first menstruation, age at first birth, and age at menopause were not significantly associated with breast cancer risk. In most previous studies, comparisons were made using categories for age at first menstruation of $12-13$ and $>15$ years old [22] and age at first birth of $\leq 24$ and $>30$ years old [23]. In the current study, the sample sizes for women who were $>15$ years old at first menstruation and $>30$ years old at first birth were too small to analyze correctly, which is a limitation in the study.

The associations of food and nutrition with breast cancer risk have been summarized by the WCRF/AICR [3]. The effects of some foods on breast cancer are unclear, but we found that intake of meat, mushrooms, yellow and green vegetables, coffee and green tea was associated with decreased breast cancer risk. The evidence that alcohol is associated with breast cancer was judged to be "convincing" by the WCRF/AICR, but we did not find this association, which is consistent with other Japanese studies. The frequency and amount of food consumption depends on cultures and customs in different countries, and this may cause the factors and threshold level for breast cancer risk to also vary in the respective countries.

Cigarette smoking $[24,25]$ is also considered to be associated with increased breast cancer risk, while leisure-time exercise [26] is associated with decreased breast cancer risk, including in the Japanese population. The mean BMI of the Asian population, including the Japanese population, is lower than that in non-Asians [27]. However, we found that $\mathrm{BMI} \geq 24$ is associated with increased breast cancer risk, as found in other Japanese studies [28].

A high education level has been associated with increased breast cancer risk, but this may be explained by highly educated women having a high rate of nulliparity and being older at first birth. However, in Japan, social advances and college attendance have only become more common for women in recent years, and thus education level may not correlate well with social status and an unwed state. Instead, more highly educated women are more likely to be involved in preventive health behavior such as exercise, non-smoking, no alcohol intake and avoidance of obesity, compared to women with less education, and some studies have associated a higher education level with a decreased breast cancer risk $[29,30]$.

The current study has several limitations. First, selection bias may have influenced the results because we enrolled women who underwent breast cancer screening as controls. In Japan, the rate of breast cancer screening was no more than about $25 \%$ in 2010 [31]. Thus, women who undergo screening may have more interest in trying to maintain their health and may have a family history of cancer, which may have eliminated the significant 
Table 1 Adjusted odds ratios and $95 \%$ confidence intervals for lifestyle factors in 472 cases and 464 controls (recruitment period: December 2010 to November 2011)

\begin{tabular}{|c|c|c|}
\hline Variables & $\begin{array}{c}\text { Case }(n=472) \\
n(\%)\end{array}$ & $\begin{array}{c}\text { Control }(n=464) \\
n(\%)\end{array}$ \\
\hline Age (year) (mean \pm SD) & $54.72 \pm 12.45$ & $53.56 \pm 11.00$ \\
\hline \multicolumn{3}{|l|}{ Menopausal status } \\
\hline Pre & 280 & 271 \\
\hline Post & 192 & 193 \\
\hline
\end{tabular}

Height $(\mathrm{cm})$

$\leq 150$
$151-155$
$156-160$
$>160$

$95 \quad$ (20) 78

78

2)

(32) $\quad 145$

$\mathrm{OR}^{\mathrm{a}}(95 \% \mathrm{Cls})$

72

(15)

81

$\begin{array}{ll}(34) & 173 \\ (24) & 118 \\ (20) & 78 \\ (22) & 93\end{array}$
1.16
Ref.
0.99
0.93

(0.78-1.71)

(0.72-1.36)

Weight (Kg)

$\begin{array}{cc}\leq 50 & 159 \\ 51-55 & 112 \\ 56-60 & 92 \\ >60 & 104\end{array}$

BMI $\left(\mathrm{Kg} / \mathrm{m}^{2}\right)$

20
$20-21.9$
$22-23.9$
$\geq 24$

(22) 96

(21)

(0.63-1.38)

Smoking status

Alcohol drinking

$$
\begin{aligned}
& \text { Never } \\
& \text { Current or former }
\end{aligned}
$$

Alcohol intake (g/day)

$$
\begin{gathered}
<5 \\
5-10
\end{gathered}
$$

$$
62
$$$$
\text { (8) }
$$$$
45
$$

Meat intake (times/week)

$$
\begin{aligned}
& \leq 1 \\
& 2-4 \\
& \geq 5
\end{aligned}
$$

$$
\text { ref. }
$$$$
1.02
$$$$
0.82
$$$$
\text { (0.75-1.39) }
$$$$
\text { (0.54-1.24) }
$$$$
0.75
$$$$
\text { (0.46-1.21) }
$$

Soy intake (times/week)

$\begin{array}{ll}\leq 1 & 45 \\ 2-4 & 236 \\ \geq 5 & 188\end{array}$

(14)

\section{7}

Ref.

0.65

(0.45-0.92)

67

Fish intake (times/week)

$$
\begin{aligned}
& \leq 1 \\
& 2-4
\end{aligned}
$$

(22)

(20)

Ref. 
Table 1 Adjusted odds ratios and $95 \%$ confidence intervals for lifestyle factors in 472 cases and 464 controls (recruitment period: December 2010 to November 2011) (Continued)

Eggs intake (times/week)

$\leq 1$
$2-4$
$\geq 5$

Milk intake (times/week)

$\leq 1$
$2-4$
$\geq 5$

Fruit intake (times/week)

$$
\begin{aligned}
& \leq 1 \\
& 2-4 \\
& \geq 5
\end{aligned}
$$

Mushrooms intake (times/week)

$$
\begin{aligned}
& \leq 1 \\
& 2-4 \\
& \geq 5
\end{aligned}
$$

Green and yellow vegetables intake (times/week)

$$
\begin{aligned}
& \leq 1 \\
& 2-4 \\
& \geq 5
\end{aligned}
$$

Coffee intake (times/week)

$$
\begin{gathered}
<1 \\
1 \\
2-3 \\
\geq 4
\end{gathered}
$$

Green tea intake (times/week)

$$
\begin{gathered}
<1 \\
1 \\
2-3 \\
\geq 4
\end{gathered}
$$

Leisure-time exercise

$$
\begin{aligned}
& \text { None } \\
& \text { Current }
\end{aligned}
$$

Intensity of physical activity ${ }^{\mathrm{b}}$ (METs/week)

$$
\begin{gathered}
0 \\
>6.0 \\
6.0-11.9 \\
12.0-23.9 \\
\geq 24.0
\end{gathered}
$$

Age at menarche (year)

$$
\begin{gathered}
\leq 12 \\
13
\end{gathered}
$$$$
\leq 14
$$

\section{8}

238

120

$\begin{array}{cc}(23) & 95 \\ (51) & 247 \\ (26) & 112\end{array}$

(21)

(25)

$\begin{array}{ll}(18) & 82 \\ (34) & 135 \\ (48) & 238\end{array}$

$\begin{array}{ll}(24) & 112 \\ (37) & 149 \\ (39) & 199\end{array}$

(34) 120

(13)

0.96

(0.66-1.41)

0.92

(0.64-1.31)

Ref.

$$
1.11
$$

(0.79-1.57)

0.86

(0.61-1.21)

(50) 204

(48)

Ref.

0.73

(0.54-0.98)

0.60

(0.40-0.91)

(40)

212

Ref.

0.66

(0.39-1.09)

0.48

(0.29-0.80)

$\begin{array}{ll}(56) & 214 \\ (11) & 42 \\ (10) & 60 \\ (11) & 80 \\ (12) & 61\end{array}$

(47)

Ref.

1.05

(47)

(31)

1.25 
Table 1 Adjusted odds ratios and 95\% confidence intervals for lifestyle factors in $\mathbf{4 7 2}$ cases and 464 controls (recruitment period: December 2010 to November 2011) (Continued)

Parity

$\begin{array}{ll}0 & 86 \\ 1-2 & 247 \\ \geq 3 & 102\end{array}$

86
247
102

(20)

(57)

(23)

75
265
107

(17)

(59)

(24)

Ref.
0.74
0.76

(0.511-1.06)

$(0.495-1.15)$

Age at first childbirth (year)

$\begin{array}{ll}<25 & 151 \\ 25-29 & 162 \\ \geq 30 & 63\end{array}$

151

$\begin{array}{ll}(40) & 142 \\ (43) & 187 \\ (17) & 50\end{array}$

142

(37)

1.22
Ref.
1.46

$(0.89-1.68)$

(49)

(0.96-2.25)

Breastfeeding

$\begin{array}{ll}\text { No } & 125 \\ \text { Yes } & 339\end{array}$

$\begin{array}{ll}(27) & 104 \\ (73) & 355\end{array}$

(77)

Ref.

0.77

$(0.57-1.04)$

History of benign breast disease

No
Yes

351
93

(79)

$(79)$
$(21)$

354
92

(88)

373
52

(79)

Ref.

1.03

(0.74-1.42)

Family history of breast cancer

$\begin{array}{lc}\text { No } & 391 \\ \text { Yes } & 53\end{array}$

History of HRT use

$\begin{array}{cc}\text { No } & 424 \\ \text { Yes } & 35\end{array}$

(12)

52

$\begin{array}{ll}(92) & 412 \\ \text { (8) } & 45\end{array}$

(88)

Ref.

0.98

(0.65-1.47)

(90) Ref.

(10) 0.76

(0.47-1.21)

Education

\begin{tabular}{lllllll} 
High school or less & 259 & $(55)$ & 196 & $(43)$ & Ref. & \\
Two-year college & 144 & $(31)$ & 144 & $(31)$ & 0.78 & $(0.57-1.05)$ \\
$\quad$ University & 64 & $(14)$ & 120 & $(26)$ & $\mathbf{0 . 4 1}$ & $\mathbf{( 0 . 2 9 - 0 . 5 9 )}$ \\
\hline
\end{tabular}

${ }^{\mathrm{a} O R}$ is adjusted for age. ${ }^{\mathrm{b}}$ Intensity of physical activity in leisure-time exercise. Significant dates are showed in boldface. OR, odds ratio; Cl, confidence interval; BMI, body mass index; HRT, hormone replacement therapy.

association of a family history of breast cancer with breast cancer risk in our study. Second, recall bias may have influenced the results because of the use of self-administered questionnaires. In particular, data from patients might lack accuracy because their answers reflected their behavior before diagnosis.

In all subjects, 3 of the 16 SNPs analyzed in the study were significantly associated with breast cancer risk. These included rs2046210 and rs3757318, which are located at $6 \mathrm{q} 25.1$, in proximity to the estrogen receptor 1 gene (ESR1). ESR1 encodes an estrogen receptor (ER $\alpha)$, a ligand-activated transcription factor composed of several domains important for hormone binding, DNA binding, and activation of transcription [32]. ER $\alpha$ is mainly expressed in the uterus, ovary, bone, and breast in females [33], ER $\alpha$ is also overexpressed in $60-70 \%$ of cases of breast cancer and is involved in the disease pathology. Although these SNPs are located in the same chromosome region, they are not in strong LD based on the HapMap Project. Potential involvement of both
SNPs in regulation of ESR1 is unclear [14,34]. rs2046210 is located $29 \mathrm{~kb}$ upstream of the first untranslated exon. The risk allele frequency of rs2046210 is 33.3\% in Europeans (HapMap-CEU), 37.8\% in Chinese (Hap MapHCB) and 30.0\% in Japanese (HapMap-JTP) [19]. Our result indicated a $27 \%$ risk allele frequency, which was about the same as that in HapMap-JTP. Thus, the risk allele frequency of Asians differs little from that of Europeans. Several studies have associated rs2046210 with breast cancer risk [15,34-36]. Guo et al. found a significant association between rs2046210 and breast cancer risk in the overall population (per allele OR 1.14, 95\% CI $=1.10-1.18$ ) and in Asians (per allele OR 1.27, 95\% $\mathrm{CI}=1.23-1.31$ ) and Europeans (per allele OR 1.09, 95\% $\mathrm{CI}=1.07-1.12$ ), indicating that rs2046210 has a larger effect in Asians [34]. Our results also suggest that rs2046210 is significantly associated with breast cancer risk in Japanese subjects.

Turnbull et al. first reported a significant association of rs3757318 with breast cancer risk [11]. rs3757318 is 


\begin{tabular}{|c|c|c|c|c|c|c|c|c|c|c|c|c|c|c|c|c|}
\hline \multirow[b]{2}{*}{$\begin{array}{l}\text { SNP } \\
\text { Gene/location }\end{array}$} & \multirow[b]{2}{*}{ Genotype $^{a}$} & \multicolumn{5}{|c|}{ All women $(n=936)$} & \multicolumn{5}{|c|}{ Premenopausal $(n=385)$} & \multicolumn{5}{|c|}{ Postmenopausal $(n=551)$} \\
\hline & & \multirow{2}{*}{$\begin{array}{c}\text { No. of } \\
\text { Case/Control } \\
7 / 4\end{array}$} & \multicolumn{2}{|c|}{$\begin{array}{c}\text { Adjusted OR }^{\mathbf{b}} \\
\text { OR }(95 \% \mathrm{Cl})\end{array}$} & \multicolumn{2}{|c|}{$\begin{array}{c}\text { Multivariate } \mathrm{OR}^{\mathrm{c}} \\
\text { OR }(95 \% \mathrm{Cl})\end{array}$} & $\begin{array}{c}\text { No. of } \\
\text { Case/Control }\end{array}$ & \multicolumn{2}{|c|}{$\begin{array}{l}\text { Adjusted OR } \\
\text { OR }(95 \% \mathrm{Cl})\end{array}$} & \multicolumn{2}{|c|}{$\begin{array}{c}\text { Multivariate } \mathrm{OR}^{\mathrm{c}} \\
\text { OR }(95 \% \mathrm{Cl})\end{array}$} & \multirow[t]{2}{*}{$\begin{array}{c}\text { No. of } \\
\text { Case/Control }\end{array}$} & \multicolumn{2}{|c|}{$\begin{array}{c}\text { Adjusted OR } \\
\text { OR }(95 \% \mathrm{Cl})\end{array}$} & \multicolumn{2}{|c|}{$\begin{array}{c}\text { Multivariate OR } \\
\text { OR }(95 \% \mathrm{Cl})\end{array}$} \\
\hline rs1562430 & CC & & Ref. & & Ref. & & $2 / 3$ & Ref. & & Ref. & & & Ref. & & Ref. & \\
\hline \multirow[t]{3}{*}{ /8q24 } & TC & $96 / 102$ & 0.54 & $(0.14-1.85)$ & 0.62 & $(0.15-2.32)$ & $33 / 42$ & 1.24 & $(0.19-9.85)$ & 1.10 & $(0.15-10.05)$ & $5 / 1$ & 0.24 & $(0.01-1.54)$ & 0.35 & $(0.02-2.80)$ \\
\hline & $\pi$ & $369 / 351$ & 0.61 & $(0.16-2.05)$ & 0.67 & $(0.16-2.45)$ & $155 / 146$ & 1.64 & $(0.27-12.63)$ & 1.72 & $(0.24-15.14)$ & $63 / 60$ & 0.24 & $(0.01-1.52)$ & 0.29 & $(0.01-2.25)$ \\
\hline & Per allele & & 1.05 & $(0.79-1.39)$ & 1.02 & $(0.75-1.39)$ & & 1.08 & $(0.81-1.45)$ & 1.62 & $(1.08-2.44)$ & $214 / 205$ & 1.07 & $(0.85-1.36)$ & 0.80 & $(0.56-1.14)$ \\
\hline rs889132 & AA & $76 / 91$ & Ref. & & Ref. & & $34 / 36$ & Ref. & & Ref. & & & Ref. & & Ref. & \\
\hline \multirow[t]{3}{*}{ MAP3K1/5q } & CA & $227 / 211$ & 1.27 & $(0.89-1.83)$ & 1.27 & $(0.86-1.88)$ & $91 / 95$ & 0.96 & $(0.55-1.65)$ & 0.82 & $(0.45-1.50)$ & $42 / 55$ & 1.59 & $(0.98-2.58)$ & 1.57 & $(0.91-2.76)$ \\
\hline & $\mathrm{CC}$ & $164 / 160$ & 1.21 & $(0.83-1.76)$ & 1.21 & $(0.81-1.81)$ & $64 / 61$ & 1.07 & $(0.60-1.92)$ & 0.98 & $(0.52-1.84)$ & $136 / 116$ & 1.35 & $(0.82-2.23)$ & 1.30 & $(0.74-2.30)$ \\
\hline & Per allele & & 1.07 & $(0.89-1.29)$ & 1.07 & $(0.88-1.31)$ & & 1.08 & $(0.81-1.45)$ & 1.11 & $(0.83-1.49)$ & 100/99 & 1.07 & $(0.85-1.36)$ & 1.05 & $(0.81-1.36)$ \\
\hline rs13283615 & $\mathrm{AA}$ & $75 / 75$ & Ref. & & Ref. & & $29 / 31$ & Ref. & & Ref. & & & ref. & & ref. & \\
\hline \multirow[t]{3}{*}{$/ 8 q 24$} & GA & $211 / 206$ & 1.04 & $(0.71-1.51)$ & 1.09 & $(0.73-1.65)$ & $73 / 80$ & 0.97 & $(0.53-1.76)$ & 1.13 & $(0.60-2.17)$ & $46 / 44$ & 1.10 & $(0.68-1.79)$ & 1.17 & $(0.67-2.05)$ \\
\hline & GG & 180/177 & 1.03 & $(0.70-1.51)$ & 1.02 & $(0.67-1.55)$ & $86 / 78$ & 1.14 & $(0.63-2.05)$ & 1.18 & $(0.62-2.24)$ & $138 / 126$ & 0.97 & $(0.58-1.61)$ & 1.09 & $(0.61-1.97)$ \\
\hline & Per allele & & 1.01 & $(0.84-1.21)$ & 1.00 & $(0.81-1.22)$ & & 1.11 & $(0.84-1.47)$ & 1.03 & $(1.00-1.05)$ & $94 / 99$ & 0.95 & $(0.74-1.21)$ & 0.99 & $(0.76-1.28)$ \\
\hline rs981782 & $\pi$ & $166 / 149$ & Ref. & & Ref. & & $67 / 64$ & Ref. & & Ref. & & & Ref. & & Ref. & \\
\hline \multirow[t]{3}{*}{ HCN1/5p12 } & TG & $220 / 234$ & 0.85 & $(0.64-1.14)$ & 0.82 & $(0.60-1.13)$ & $88 / 98$ & 0.85 & $(0.54-1.33)$ & 0.78 & $(0.48-1.26)$ & $99 / 85$ & 0.87 & $(0.59-1.27)$ & 0.83 & $(0.54-1.29)$ \\
\hline & GG & $82 / 76$ & 0.96 & $(0.66-1.41)$ & 0.88 & $(0.58-1.34)$ & $31 / 28$ & 1.03 & $(0.56-1.91)$ & 0.97 & $(0.50-1.90)$ & $132 / 136$ & 0.93 & $(0.57-1.52)$ & 0.76 & $(0.43-1.34)$ \\
\hline & Per allele & & 0.95 & (0.79-1.14) & 0.97 & $(0.80-1.17)$ & & 1.00 & $(0.75-1.35)$ & 1.01 & $(0.74-1.38)$ & $51 / 48$ & 0.93 & $(0.73-1.18)$ & 0.86 & $(0.66-1.13)$ \\
\hline rs3803662 & $C C$ & $74 / 91$ & Ref. & & Ref. & & $24 / 42$ & Ref. & & Ref. & & & Ref. & & Ref. & \\
\hline \multirow[t]{3}{*}{ TNRC9/16q12 } & TC & $230 / 227$ & 1.25 & $(0.88-1.79)$ & 1.32 & $(0.89-1.96)$ & $89 / 96$ & 1.59 & $(0.90-2.85)$ & 1.50 & $(0.81-2.80)$ & $50 / 49$ & 1.08 & $(0.68-1.72)$ & 1.25 & $(0.73-2.16)$ \\
\hline & $\pi$ & $160 / 142$ & 1.41 & $(0.97-2.08)$ & 1.61 & $(1.06-2.45)$ & $72 / 53$ & 2.29 & $(1.25-4.26)$ & 2.29 & $(1.20-4.46)$ & $141 / 131$ & 1.04 & $(0.63-1.71)$ & 1.27 & $(0.72-2.24)$ \\
\hline & Per allele & & 1.18 & (0.98-1.42) & 1.28 & $(1.07-1.55)$ & & 1.54 & $(1.15-2.09)$ & 1.58 & $(1.17-2.16)$ & $88 / 89$ & 1.00 & $(0.78-1.28)$ & 1.07 & $(0.83-1.39)$ \\
\hline rs381798 & $\pi$ & $339 / 347$ & Ref. & & Ref. & & $138 / 140$ & Ref. & & Ref. & & & Ref. & & Ref. & \\
\hline \multirow[t]{3}{*}{ LSP1/11p15.5 } & CT & 120/107 & 1.14 & $(0.85-1.55)$ & 1.07 & $(0.77-1.49)$ & $46 / 49$ & 0.92 & $(0.58-1.48)$ & 1.00 & $(0.60-1.68)$ & $201 / 207$ & 1.30 & $(0.87-1.94)$ & 1.18 & $(0.75-1.86)$ \\
\hline & CC & $10 / 5$ & 2.04 & $(0.72-6.60)$ & 1.63 & $(0.52-5.66)$ & $4 / 1$ & 3.98 & $(0.58-78.39)$ & 3.29 & $(0.42-68.89)$ & $74 / 58$ & 1.65 & $(0.46-6.55)$ & 1.39 & $(0.32-6.31)$ \\
\hline & Per allele & & 1.19 & $(0.91-1.56)$ & 1.11 & $(0.83-1.49)$ & & 1.07 & $(0.70-1.64)$ & 1.21 & $(0.77-1.90)$ & $6 / 4$ & 1.27 & $(0.90-1.81)$ & 1.14 & $(0.78-1.66)$ \\
\hline rs2046210 & GG & $213 / 244$ & Ref. & & Ref. & & $83 / 107$ & Ref. & & Ref. & & & Ref. & & Ref. & \\
\hline \multirow[t]{3}{*}{ ESR1/6q25.1 } & $A G$ & 194/185 & 1.21 & $(0.92-1.59)$ & 1.22 & $(0.90-1.64)$ & 78/72 & 1.41 & $(0.92-2.17)$ & 1.63 & $(1.03-2.61)$ & 130/137 & 1.11 & $(0.78-1.59)$ & 0.99 & $(0.67-1.48)$ \\
\hline & AA & $61 / 34$ & 2.03 & $(1.29-3.25)$ & 2.16 & $(1.32-3.59)$ & $27 / 14$ & 2.46 & $(1.23-5.10)$ & 2.93 & $(1.40-6.40)$ & $116 / 113$ & 1.69 & $(0.93-3.14)$ & 1.69 & $(0.84-3.50)$ \\
\hline & Per allele & & 1.34 & $(1.10-1.63)$ & 1.37 & $(1.11-1.70)$ & & 1.49 & $(1.10-2.03)$ & 1.70 & $(1.24-2.35)$ & $34 / 20$ & 1.23 & $(0.95-1.59)$ & 1.14 & $(0.86-1.51)$ \\
\hline
\end{tabular}


Table 2 Odds ratio with $95 \%$ confidence intervals for individual SNPs in all subjects and in subjects stratified by menopausal status (Continued)

\begin{tabular}{|c|c|c|c|c|c|c|c|c|c|c|c|c|c|c|c|c|}
\hline rs909116 & CC & $166 / 178$ & Ref. & & Ref. & & $71 / 64$ & Ref. & & Ref. & & & Ref. & & Ref. & \\
\hline \multirow[t]{3}{*}{ LSP/11p15.5 } & CT & $225 / 228$ & 1.08 & $(0.81-1.43)$ & 1.04 & $(0.77-1.42)$ & $88 / 106$ & 0.76 & $(0.49-1.18)$ & 0.90 & $(0.55-1.47)$ & $95 / 114$ & 1.36 & $(0.94-1.97)$ & 1.20 & $(0.79-1.83)$ \\
\hline & $\pi$ & $79 / 57$ & 1.49 & $(0.99-2.24)$ & 1.40 & $(0.90-2.19)$ & $30 / 23$ & 1.21 & $(0.64-2.30)$ & 1.23 & $(0.62-2.48)$ & $137 / 122$ & 1.72 & $(1.02-2.90)$ & 1.69 & $(0.94-3.09)$ \\
\hline & Per allele & & 1.18 & $(0.97-1.42)$ & 1.15 & $(0.93-1.41)$ & & 0.98 & $(0.72-1.32)$ & 1.11 & $(0.81-1.52)$ & $49 / 34$ & 1.32 & $(1.03-1.69)$ & 1.24 & $(0.95-1.63)$ \\
\hline rs30099 & CC & $225 / 216$ & Ref. & & Ref. & & $93 / 84$ & Ref. & & Ref. & & & Ref. & & Ref. & \\
\hline \multirow[t]{3}{*}{$15 q$} & TC & 205/198 & 0.82 & $(0.52-1.29)$ & 1.08 & $(0.80-1.45)$ & $82 / 84$ & 0.87 & $(0.57-1.33)$ & 0.96 & $(0.61-1.53)$ & $132 / 132$ & 1.08 & $(0.76-1.54)$ & 1.21 & $(0.80-1.83)$ \\
\hline & $\pi$ & $42 / 50$ & 0.99 & $(0.76-1.30)$ & 0.86 & $(0.52-1.41)$ & $15 / 25$ & 0.53 & $(0.26-1.06)$ & 0.51 & $(0.24-1.08)$ & $123 / 114$ & 1.12 & $(0.61-2.06)$ & 1.19 & $(0.58-2.45)$ \\
\hline & Per allele & & 0.93 & $(0.76-1.13)$ & 0.98 & $(0.79-1.22)$ & & 0.78 & $(0.57-1.06)$ & 0.85 & $(0.92-1.16)$ & $27 / 25$ & 1.04 & $(0.81-1.36)$ & 1.12 & $(0.83-1.50)$ \\
\hline rs2981282 & CC & $220 / 226$ & Ref. & & Ref. & & $86 / 94$ & Ref. & & Ref. & & & Ref. & & Ref. & \\
\hline \multirow[t]{3}{*}{ FGFR2 /10q26 } & TC & $210 / 190$ & 1.15 & $(0.87-1.50)$ & 1.19 & $(0.89-1.60)$ & $91 / 81$ & 1.23 & $(0.81-1.87)$ & 1.48 & $(0.94-2.35)$ & $134 / 132$ & 1.10 & (0.77-1.58) & 1.08 & $(0.72-1.62)$ \\
\hline & $\pi$ & $41 / 45$ & 0.92 & $(0.58-1.47)$ & 0.84 & $(0.50-1.40)$ & $13 / 17$ & 0.89 & $(0.41-1.92)$ & 1.07 & $(0.46-2.50)$ & $119 / 109$ & 0.95 & $(0.53-1.71)$ & 0.76 & $(0.38-1.48)$ \\
\hline & Per allele & & 1.03 & $(0.84-1.25)$ & 1.02 & $(0.82-1.27)$ & & 1.04 & $(0.75-1.43)$ & 1.27 & $(0.91-1.78)$ & $28 / 28$ & 1.04 & $(0.80-1.34)$ & 0.94 & $(0.71-1.24)$ \\
\hline rs795399 & $\pi$ & $255 / 249$ & Ref. & & Ref. & & $90 / 107$ & Ref. & & Ref. & & & Ref. & & Ref. & \\
\hline \multirow[t]{3}{*}{ IGF1/12q23.2 } & $\mathrm{CT}$ & $180 / 173$ & 0.84 & $(0.51-1.36)$ & 1.05 & $(0.78-1.41)$ & $82 / 65$ & 1.49 & $(0.97-2.30)$ & 1.56 & $(0.98-2.48)$ & $165 / 142$ & 0.80 & $(0.56-1.15)$ & 0.78 & $(0.52-1.18)$ \\
\hline & CC & $34 / 41$ & 1.03 & $(0.78-1.35)$ & 0.85 & $(0.49-1.45)$ & $15 / 20$ & 0.86 & $(0.41-1.77)$ & 1.04 & $(0.46-2.27)$ & $98 / 108$ & 0.87 & $(0.44-1.70)$ & 0.93 & $(0.43-1.99)$ \\
\hline & Per allele & & 0.96 & $(0.79-1.18)$ & 0.97 & $(0.78-1.21)$ & & 1.13 & $(0.83-1.55)$ & 1.25 & $(0.91-1.72)$ & $19 / 21$ & 0.87 & $(0.66-1.14)$ & 0.88 & $(0.66-1.17)$ \\
\hline rs3757318 & GG & $249 / 281$ & Ref. & & Ref. & & $95 / 111$ & Ref. & & Ref. & & & Ref. & & Ref. & \\
\hline \multirow[t]{3}{*}{ ESR1/6q25.1 } & $A G$ & $182 / 162$ & 1.27 & $(0.97-1.67)$ & 1.25 & $(0.93-1.69)$ & $76 / 72$ & 1.25 & $(0.82-1.91)$ & 1.22 & $(0.77-1.92)$ & $154 / 170$ & 1.27 & (0.88-1.81) & 1.20 & $(0.79-1.80)$ \\
\hline & $A A$ & $34 / 19$ & 2.01 & $(1.13-3.68)$ & 2.05 & $(1.09-3.97)$ & $14 / 8$ & 2.02 & $(0.83-5.25)$ & 1.90 & $(0.73-5.25)$ & $106 / 90$ & 1.96 & $(0.92-4.37)$ & 2.14 & $(0.88-5.49)$ \\
\hline & Per allele & & 1.34 & $(1.08-1.66)$ & 1.33 & $(1.05-1.69)$ & & 1.30 & $(0.93-1.83)$ & 1.34 & $(0.95-1.91)$ & $20 / 11$ & 1.32 & $(1.00-1.76)$ & 1.27 & $(0.93-1.75)$ \\
\hline
\end{tabular}

aAlleles on upper line are common alleles; ${ }^{\mathrm{b}}$ Adjusted for age; ${ }^{\mathrm{C}}$ Multivariate adjusted for age, BMl, smoking, meat intake, mushroom intake, green and yellow vegetable intake, coffee intake, green tea intake, leisure-time exercise and education. Significant dates are showed in boldface. $\mathrm{OR}$, odds ratio; $\mathrm{Cl}$, confidence interval. 
Table 3 Age-adjusted odds ratio and multivariate adjusted odds ratio with $95 \%$ confidence intervals for lifestyle factors in rs 2046210

\begin{tabular}{|c|c|c|c|c|c|c|c|c|c|c|c|c|c|c|c|}
\hline & & \multicolumn{7}{|c|}{$\begin{array}{l}\text { Risk allele carriers (AA }+A G) n=474 \\
\quad \text { Case } n=255 / \text { Control } n=219\end{array}$} & \multicolumn{7}{|c|}{$\begin{array}{c}\text { Non-risk allele carriers (GG) } n=457 \\
\text { Case } n=213 / \text { Control } n=244\end{array}$} \\
\hline & & \multirow{2}{*}{$\begin{array}{c}\mathbf{n} / \mathbf{n} \\
54.0 / 53.9\end{array}$} & \multicolumn{2}{|c|}{$\mathrm{OR}^{\mathrm{a}}(95 \% \mathrm{Cl})$} & \multirow[t]{2}{*}{$p$} & \multicolumn{2}{|c|}{$\mathrm{OR}^{\mathrm{b}}(95 \% \mathrm{CI})$} & \multirow[t]{2}{*}{$\mathrm{p}$} & \multirow{2}{*}{$\begin{array}{c}\mathbf{n} / \mathbf{n} \\
55.8 / 53.2\end{array}$} & \multicolumn{2}{|c|}{$\mathrm{OR}^{\mathrm{a}}(95 \% \mathrm{Cl})$} & \multirow[t]{2}{*}{$p$} & \multicolumn{2}{|c|}{ OR $^{c}(95 \% \mathrm{Cl})$} & \multirow[t]{2}{*}{$p$} \\
\hline Age (years) & & & & & & & & & & & & & & & \\
\hline \multirow[t]{2}{*}{ Menopausal status } & Pre & $148 / 133$ & & & & & & & 130/137 & & & & & & \\
\hline & Post & 107/86 & & & & & & & $83 / 107$ & & & & & & \\
\hline \multirow[t]{4}{*}{ Height (cm) } & $\leq 150$ & $40 / 39$ & 1.03 & $(0.58-1.83)$ & 0.93 & 0.96 & $(0.53-1.74)$ & 0.89 & $55 / 39$ & 1.34 & $(0.78-2.9)$ & 0.29 & 1.19 & $(0.66-2.14)$ & 0.57 \\
\hline & $151-155$ & $76 / 77$ & Ref. & & & Ref. & & & $68 / 68$ & Ref. & & & Ref. & & \\
\hline & $156-160$ & $89 / 66$ & 1.38 & $(0.88-2.16)$ & 0.16 & 1.44 & $(0.91-2.29)$ & 0.12 & $63 / 89$ & 0.76 & $(0.48-1.3)$ & 0.27 & 0.89 & $(0.53-1.48)$ & 0.64 \\
\hline & $>160$ & $46 / 34$ & 1.41 & $(0.81-2.47)$ & 0.23 & 1.62 & $(0.91-2.91)$ & 0.10 & $25 / 47$ & 0.59 & $(0.32-1.08)$ & 0.09 & 0.51 & $(0.25-0.99)$ & 0.05 \\
\hline \multirow[t]{4}{*}{$\mathrm{BMI}\left(\mathrm{Kg} / \mathrm{m}^{2}\right)$} & 20 & $59 / 46$ & 1.27 & $(0.75-2.14)$ & 0.37 & 1.13 & $(0.67-1.94)$ & 0.64 & $43 / 50$ & 1.62 & $(0.93-2.81)$ & 0.09 & 1.54 & $(0.84-2.82)$ & 0.16 \\
\hline & $20-21.9$ & $69 / 67$ & Ref. & & & Ref. & & & $48 / 82$ & Ref. & & & Ref. & & \\
\hline & $22-23.9$ & $58 / 50$ & 1.09 & $(0.66-1.80)$ & 0.75 & 0.97 & $(0.58-1.63)$ & 0.92 & $43 / 52$ & 1.40 & $(0.82-2.40)$ & 0.22 & 1.47 & $(0.83-2.63)$ & 0.19 \\
\hline & $\geq 24$ & $65 / 53$ & 1.17 & $(0.71-1.94)$ & 0.53 & 1.09 & $(0.65-1.82)$ & 0.74 & $74 / 59$ & 2.07 & $(1.26-3.43)$ & $<0.01$ & 1.91 & $(1.11-3.29)$ & 0.02 \\
\hline \multirow[t]{2}{*}{ Smoking status } & Never & $222 / 201$ & Ref. & & & Ref. & & & $180 / 230$ & Ref. & & & Ref. & & \\
\hline & Current or former & 29/15 & 1.78 & $(0.93-3.51)$ & 0.08 & 1.61 & $(0.83-3.21)$ & 0.16 & $31 / 13$ & 3.82 & $(1.94-7.98)$ & $<0.01$ & 3.86 & $(1.87-8.37)$ & $<0.01$ \\
\hline \multirow[t]{2}{*}{ Alcohol drinking } & Never & 129/107 & Ref. & & & Ref. & & & 108/111 & Ref. & & & Ref. & & \\
\hline & Current or former & $125 / 109$ & 0.97 & $(0.67-1.40)$ & 0.97 & 1.07 & $(0.73-1.57)$ & 0.74 & 105/133 & 0.91 & $(0.62-1.33)$ & 0.61 & 0.87 & $(0.56-1.33)$ & 0.51 \\
\hline \multirow[t]{4}{*}{ Alcohol intake (g/day) } & 0 & 129/107 & Ref. & & & Ref. & & & 108/111 & Ref. & & & Ref. & & \\
\hline & $<5$ & $75 / 56$ & 1.12 & $(0.72-1.74)$ & 0.61 & 1.22 & $(0.78-1.92)$ & 0.39 & $64 / 73$ & 0.99 & $(0.64-1.54)$ & 0.98 & 0.98 & $(0.60-1.61)$ & 0.94 \\
\hline & $5-10$ & $28 / 32$ & 0.75 & $(0.42-1.34)$ & 0.34 & 0.88 & $(0.49-1.60)$ & 0.68 & $25 / 30$ & 0.94 & $(0.51-1.72)$ & 0.85 & 0.92 & $(0.46-1.80)$ & 0.80 \\
\hline & $10>$ & $20 / 19$ & 0.88 & $(0.44-1.74)$ & 0.71 & 0.94 & $(0.46-1.89)$ & 0.85 & $16 / 26$ & 0.70 & $(0.35-1.38)$ & 0.31 & 0.55 & $(0.24-1.22)$ & 0.14 \\
\hline \multirow[t]{2}{*}{ Leisure-time exercise } & No & $143 / 97$ & Ref. & & & Ref. & & & $110 / 116$ & Ref. & & & Ref. & & \\
\hline & Yes & $110 / 121$ & 0.62 & $(0.43-0.89)$ & 0.01 & 0.60 & $(0.41-0.87)$ & $<0.01$ & 101/127 & 0.77 & $(0.52-1.12)$ & 0.17 & 0.74 & $(0.49-1.11)$ & 0.14 \\
\hline \multirow[t]{5}{*}{ Intensity of physical activity ${ }^{\text {d }}$ (met/week) } & 0 & $143 / 99$ & Ref. & & & Ref. & & & 109/119 & Ref. & & & Ref. & & \\
\hline & $>6.0$ & $25 / 23$ & 0.79 & $(0.42-1.48)$ & 0.45 & 0.72 & $(0.38-1.37)$ & 0.32 & $25 / 19$ & 1.35 & $(0.70-2.63)$ & 0.37 & 1.20 & $(0.59-2.48)$ & 0.61 \\
\hline & $6.0-11.9$ & $20 / 28$ & 0.49 & $(0.26-0.92)$ & 0.03 & 0.46 & $(0.24-0.86)$ & 0.02 & $22 / 32$ & 0.63 & $(0.34-1.17)$ & 0.15 & 0.66 & $(0.34-1.28)$ & 0.22 \\
\hline & $12.0-23.9$ & $27 / 36$ & 0.52 & $(0.29-0.91)$ & 0.02 & 0.53 & $(0.30-0.94)$ & 0.03 & $21 / 44$ & 0.48 & $(0.26-0.85)$ & 0.01 & 0.45 & $(0.24-0.83)$ & 0.01 \\
\hline & $\geq 24.0$ & $30 / 32$ & 0.65 & $(0.37-1.14)$ & 0.13 & 0.68 & $(0.38-1.20)$ & 0.18 & $22 / 29$ & 0.74 & (0.40-1.38) & 0.35 & 0.70 & $(0.36-1.36)$ & 0.30 \\
\hline Age at menarche & $\leq 12$ & 70/92 & 0.73 & $(0.45-1.19)$ & 0.73 & 0.72 & (0.44-1.19) & 0.20 & 68/109 & 1.07 & $(0.63-1.81)$ & 0.80 & 0.98 & $(0.56-1.70)$ & 0.93 \\
\hline \multirow[t]{2}{*}{ (year) } & 13 & $66 / 55$ & Ref. & & & Ref. & & & $43 / 58$ & Ref. & & & Ref. & & \\
\hline & $\leq 14$ & $116 / 68$ & 1.20 & $(0.74-1.93)$ & 1.20 & 1.15 & $(0.71-1.89)$ & 0.57 & $99 / 75$ & 1.32 & $(0.78-2.25)$ & 0.29 & 1.62 & $(0.93-2.84)$ & 0.09 \\
\hline
\end{tabular}


Table 3 Age-adjusted odds ratio and multivariate adjusted odds ratio with $95 \%$ confidence intervals for lifestyle factors in rs 2046210 (Continued)

\begin{tabular}{|c|c|c|c|c|c|c|c|c|c|c|c|c|c|c|c|}
\hline \multirow[t]{3}{*}{ Parity } & 0 & $54 / 35$ & Ref. & & & Ref. & & & $31 / 40$ & Ref. & & & Ref. & & \\
\hline & $1-2$ & $123 / 122$ & 0.63 & $(0.38-1.04)$ & 0.07 & 0.66 & $(0.40-1.10)$ & 0.11 & $124 / 143$ & 0.95 & $(0.55-1.64)$ & 0.85 & 1.12 & $(0.61-2.09)$ & 0.71 \\
\hline & $\geq 3$ & $54 / 53$ & 0.65 & $(0.36-1.15)$ & 0.14 & 0.65 & $(0.36-1.17)$ & 0.15 & $46 / 53$ & 0.94 & $(0.50-1.76)$ & 0.84 & 1.29 & $(0.64-2.62)$ & 0.48 \\
\hline Age at first childbirth & $<25$ & $78 / 68$ & 1.21 & $(0.77-1.90)$ & 0.40 & 1.08 & $(0.68-1.71)$ & 0.74 & $72 / 74$ & 1.22 & $(0.78-1.91)$ & 0.38 & 1.17 & $(0.71-1.91)$ & 0.54 \\
\hline \multirow[t]{2}{*}{ (year) } & $25-29$ & $87 / 89$ & Ref. & & & Ref. & & & $75 / 97$ & Ref. & & & Ref. & & \\
\hline & $\geq 30$ & $33 / 22$ & 1.55 & $(0.84-2.90)$ & 0.16 & 1.45 & $(0.77-2.76)$ & 0.25 & $30 / 28$ & 1.39 & $(0.77-2.54)$ & 0.27 & 1.77 & $(0.92-3.45)$ & 0.09 \\
\hline \multirow[t]{2}{*}{ Breastfeeding } & No & $72 / 51$ & Ref. & & & Ref. & & & $51 / 53$ & Ref. & & & Ref. & & \\
\hline & Yes & $178 / 165$ & 0.76 & $(0.50-1.16)$ & 0.21 & 0.77 & $(0.50-1.17)$ & 0.22 & 159/189 & 0.83 & $(0.53-1.30)$ & 0.42 & 1.02 & $(0.62-1.69)$ & 0.93 \\
\hline Family history of & No & 209/180 & Ref. & & & Ref. & & & $178 / 192$ & Ref. & & & Ref. & & \\
\hline Breast cancer & Yes & $31 / 24$ & 1.11 & $(0.63-1.97)$ & 0.55 & 1.12 & $(0.63-2.00)$ & 0.71 & $22 / 28$ & 0.82 & $(0.45-1.50)$ & 0.75 & 1.07 & $(0.57-2.05)$ & 0.83 \\
\hline \multirow[t]{3}{*}{ Education } & High school or less & 135/99 & Ref. & & & Ref. & & & $123 / 96$ & Ref. & & & Ref. & & \\
\hline & Two-year college & $81 / 63$ & 0.93 & $(0.61-1.42)$ & 0.74 & 0.95 & $(0.62-1.47)$ & 0.83 & $60 / 81$ & 0.62 & $(0.40-0.95)$ & 0.03 & 0.59 & $(0.37-0.94)$ & 0.03 \\
\hline & University & $36 / 55$ & 0.48 & $(0.29-0.79)$ & $<0.01$ & 0.48 & $(0.29-0.79)$ & $<0.01$ & $28 / 65$ & 0.35 & $(0.21-0.59)$ & $<0.01$ & 0.38 & $(0.22-0.66)$ & $<0.01$ \\
\hline
\end{tabular}

${ }^{\mathrm{a} O R}$ is adjusted for age.

${ }^{\mathrm{b}}$ Multivariate adjusted for leisure-time exercise and education

'Multivariate adjusted for BMI, smoking state, intensity of physical activity and education.

dIntensity of physical activity and education. Significant dates are showed in boldface.

$\mathrm{OR}$, odds ratio; $\mathrm{Cl}$, confidence interval; $\mathrm{BMI}$, body mass index. 


\begin{tabular}{|c|c|c|c|c|c|c|c|c|c|c|c|c|c|c|c|}
\hline \multirow[b]{3}{*}{ Age (years) } & & \multicolumn{7}{|c|}{$\begin{array}{l}\text { Risk allele carriers }(A A+A G) n=397 \\
\text { Case } n=216 / \text { Control } n=181\end{array}$} & \multicolumn{7}{|c|}{$\begin{array}{c}\text { non-risk allele carriers }(G G) n=530 \\
\text { Case } n=249 / \text { Control } n=281\end{array}$} \\
\hline & & \multirow{2}{*}{$\begin{array}{c}\mathbf{n} / \mathbf{n} \\
54.23 / 53.30\end{array}$} & \multicolumn{2}{|c|}{$\mathrm{OR}^{\mathrm{a}}(95 \% \mathrm{Cl})$} & \multirow[t]{2}{*}{$p$} & \multicolumn{2}{|c|}{$\mathrm{OR}^{\mathrm{b}}(95 \% \mathrm{Cl})$} & \multirow[t]{2}{*}{$p$} & \multirow{2}{*}{$\begin{array}{c}\mathbf{n} / \mathbf{n} \\
55.28 / 53.76\end{array}$} & \multicolumn{2}{|c|}{$\mathrm{OR}^{\mathrm{a}}(95 \% \mathrm{Cl})$} & \multirow[t]{2}{*}{$p$} & \multicolumn{2}{|c|}{$\mathrm{OR}^{\mathrm{c}}(95 \% \mathrm{Cl})$} & \multirow[t]{2}{*}{$p$} \\
\hline & & & & & & & & & & & & & & & \\
\hline \multirow[t]{2}{*}{ Menopausal status } & Pre & $124 / 101$ & & & & & & & $154 / 170$ & & & & & & \\
\hline & Post & $92 / 80$ & & & & & & & $95 / 111$ & & & & & & \\
\hline \multirow[t]{4}{*}{ Height (cm) } & $\leq 150$ & $36 / 28$ & 1.24 & $(0.66-2.34)$ & 0.50 & 1.46 & $(0.68-3.16)$ & 0.33 & $58 / 50$ & 1.07 & $(0.65-1.77)$ & 0.78 & 1.01 & $(0.60-1.69)$ & 0.98 \\
\hline & $151-155$ & $62 / 63$ & Ref. & & & Ref. & & & $84 / 80$ & Ref. & & & ref. & & \\
\hline & $156-160$ & $78 / 51$ & 1.57 & $(0.96-260)$ & 0.07 & 1.57 & $(0.86-2.90)$ & 0.14 & $72 / 105$ & 0.68 & (0.44-1.05) & 0.08 & 0.73 & $(0.47-1.15)$ & 0.18 \\
\hline & $>160$ & $36 / 38$ & 1.00 & $(0.55-1.80)$ & 0.99 & 0.58 & $(0.26-1.24)$ & 0.16 & $34 / 43$ & 0.80 & $(0.46-1.39)$ & 0.43 & 0.89 & $(0.50-1.59)$ & 0.70 \\
\hline \multirow[t]{4}{*}{$\mathrm{BMI}\left(\mathrm{Kg} / \mathrm{m}^{2}\right)$} & $<20$ & $48 / 37$ & 1.36 & $(0.77-2.40)$ & 0.26 & 1.11 & $(0.54-2.29)$ & 0.77 & $54 / 59$ & 1.57 & $(0.95-2.59)$ & 0.06 & 1.60 & $(0.95-2.69)$ & 0.08 \\
\hline & $20-21.9$ & $59 / 60$ & Ref. & & & Ref. & & & $54 / 90$ & Ref. & & & Ref. & & \\
\hline & $22-23.9$ & $47 / 35$ & 1.35 & $(0.77-2.40)$ & 0.24 & 1.57 & $(0.80-3.12)$ & 0.19 & $57 / 66$ & 1.41 & $(0.86-2.30)$ & 0.40 & 1.29 & $(0.78-2.14)$ & 0.32 \\
\hline & $\geq 24$ & $57 / 48$ & 1.18 & $(0.69-2.01)$ & 0.51 & 1.14 & $(0.60-2.17)$ & 0.68 & $81 / 63$ & 2.08 & $3 \quad(1.29-3.37)$ & $<0.01$ & 1.89 & $(1.16-3.10)$ & 0.01 \\
\hline \multirow[t]{2}{*}{ Smoking status } & Never & 186/168 & Ref. & & & Ref. & & & $214 / 262$ & Ref. & & & Ref. & & \\
\hline & Current or former & $25 / 11$ & 2.15 & $(1.05-4.71)$ & 0.04 & 2.73 & $(1.07-7.65)$ & 0.04 & $34 / 17$ & 2.82 & $2(1.53-5.40)$ & $<0.01$ & 2.39 & $(1.27-4.65)$ & $<0.01$ \\
\hline \multirow[t]{2}{*}{ Alcohol drinking } & Never & $114 / 90$ & Ref. & & & Ref. & & & $124 / 127$ & Ref. & & & Ref. & & \\
\hline & Current or former & $101 / 89$ & 0.93 & $(0.62-1.39)$ & 0.71 & 0.99 & $(0.60-1.65)$ & 0.97 & $125 / 153$ & 0.90 & $(0.63-1.28)$ & 0.55 & 0.95 & $(0.65-1.38)$ & 0.78 \\
\hline Alcohol intake & 0 & $114 / 90$ & Ref. & & & Ref. & & & $124 / 127$ & Ref. & & & Ref. & & \\
\hline \multirow[t]{3}{*}{ (g/day) } & $<5$ & $59 / 45$ & 1.08 & $(0.67-1.76)$ & 0.75 & 1.12 & $(0.61-2.04)$ & 0.72 & $78 / 84$ & 1.01 & $(0.67-1.51)$ & 0.98 & 1.11 & $(0.72-1.70)$ & 0.64 \\
\hline & $5-10$ & $27 / 27$ & 0.81 & $(0.44-1.49)$ & 0.50 & 0.88 & $(0.41-1.90)$ & 0.75 & $25 / 35$ & 0.79 & $(0.44-1.41)$ & 0.43 & 0.89 & $(0.49-1.63)$ & 0.71 \\
\hline & $10>$ & $13 / 16$ & 0.65 & $(0.29-1.41)$ & 0.27 & 0.78 & $(0.27-2.14)$ & 0.63 & $22 / 29$ & 0.82 & $(0.44-1.52)$ & 0.54 & 0.66 & $(0.33-1.28)$ & 0.22 \\
\hline Leisure-time & No & $122 / 80$ & Ref. & & & Ref. & & & $127 / 133$ & Ref. & & & Ref. & & \\
\hline Exercise & Yes & $93 / 101$ & 0.58 & $(0.39-0.87)$ & $<0.01$ & 0.78 & $(0.47-1.27)$ & 0.32 & 119/146 & 0.82 & $(0.58-1.17)$ & 0.27 & 0.84 & $(0.59-1.21)$ & 0.35 \\
\hline \multirow[t]{5}{*}{ Intensity of physical activity ${ }^{d}$ (met/week) } & 0 & $122 / 81$ & Ref. & & & Ref. & & & $126 / 137$ & Ref. & & & Ref. & & \\
\hline & $>6.0$ & 23/17 & 0.87 & $(0.44-1.76)$ & 0.70 & 1.62 & $(0.68-4.03)$ & 0.28 & $28 / 25$ & 1.24 & $(0.68-2.27)$ & 0.48 & 1.19 & $(0.64-2.25)$ & 0.58 \\
\hline & $6.0-11.9$ & $21 / 25$ & 0.55 & $(0.28-1.04)$ & 0.07 & 0.58 & $(0.27-1.21)$ & 0.15 & $23 / 34$ & 0.68 & $(0.37-1.22)$ & 0.20 & 0.69 & $(0.37-1.28)$ & 0.24 \\
\hline & $12.0-23.9$ & $19 / 32$ & 0.39 & $(0.20-0.73)$ & $<0.01$ & 0.73 & $(0.33-1.56)$ & 0.41 & $29 / 48$ & 0.63 & $(0.37-1.06)$ & 0.08 & 0.62 & $(0.36-1.06)$ & 0.08 \\
\hline & $\geq 24.0$ & $23 / 26$ & 0.56 & $(0.29-1.06)$ & 0.07 & 0.67 & $(0.31-1.42)$ & 0.29 & $27 / 35$ & 0.79 & $(0.45-1.39)$ & 0.42 & 0.84 & $(0.47-1.50)$ & 0.55 \\
\hline Age at menarche & $\leq 12$ & $63 / 73$ & 1.00 & $(0.59-1.70)$ & 0.99 & 1.67 & $(0.85-3.33)$ & 0.14 & $73 / 127$ & 0.77 & $(0.47-1.24)$ & 0.28 & 0.74 & $(0.45-1.22)$ & 0.24 \\
\hline \multirow[t]{2}{*}{ (year) } & 13 & $52 / 51$ & Ref. & & & Ref. & & & $57 / 61$ & Ref. & & & Ref. & & \\
\hline & $\leq 14$ & $99 / 56$ & 1.39 & $(0.82-2.35)$ & 0.22 & 1.74 & $(0.90-3.37)$ & 0.10 & $115 / 88$ & 1.12 & $(0.70-1.81)$ & 0.63 & 1.02 & $(0.62-1.68)$ & 0.92 \\
\hline
\end{tabular}


Table 4 Age-adjusted odds ratio and multivariate adjusted odds ratio with $95 \%$ confidence intervals for lifestyle factors in rs 3757318 (Continued)

\begin{tabular}{|c|c|c|c|c|c|c|c|c|c|c|c|c|c|c|c|}
\hline \multirow[t]{3}{*}{ Parity } & 0 & $49 / 24$ & Ref. & & & Ref. & & & $37 / 50$ & Ref. & & & Ref. & & \\
\hline & $1-2$ & 110/105 & 0.48 & $(0.27-0.84)$ & $<0.01$ & 0.55 & $(0.19-1.54)$ & 0.25 & $132 / 160$ & 0.98 & $(0.60-1.62)$ & 0.95 & 1.19 & $(0.70-2.05)$ & 0.52 \\
\hline & $\geq 3$ & $36 / 48$ & 0.34 & $(0.17-0.65)$ & $<0.01$ & 0.35 & $(0.12-1.04)$ & 0.06 & $65 / 58$ & 1.36 & $(0.77-2.40)$ & 0.29 & 1.74 & $(0.95-3.21)$ & 0.07 \\
\hline Age at first childbirth & $<25$ & $60 / 60$ & 1.05 & $(0.64-1.71)$ & 0.86 & 0.97 & $(0.56-1.66)$ & 0.90 & $88 / 82$ & 1.35 & $(0.89-2.05)$ & 0.15 & 1.19 & $(0.77-1.84)$ & 0.43 \\
\hline \multirow[t]{2}{*}{ (year) } & $25-29$ & $72 / 77$ & & & & & & & $88 / 110$ & Ref. & & & Ref. & & \\
\hline & $\geq 30$ & $34 / 19$ & 1.96 & $(1.03-3.80)$ & 0.04 & 1.82 & $(0.88-3.85)$ & 0.11 & 29/31 & 1.17 & $(0.66-2.10)$ & 0.59 & 1.27 & $(0.69-2.33)$ & 0.45 \\
\hline \multirow[t]{2}{*}{ Breastfeeding } & No & $65 / 38$ & Ref. & & & Ref. & & & $59 / 65$ & Ref. & & & Ref. & & \\
\hline & Yes & $150 / 143$ & 0.60 & $(0.38-0.95)$ & 0.03 & 0.93 & $(0.36-2.43)$ & 0.89 & 183/211 & 0.91 & $(0.61-1.38)$ & 0.67 & 1.07 & $(0.69-1.65)$ & 0.77 \\
\hline Family history of & No & $173 / 143$ & Ref. & & & Ref. & & & $212 / 229$ & Ref. & & & Ref. & & \\
\hline Breast cancer & Yes & $24 / 19$ & 1.04 & $(0.55-2.00)$ & 0.79 & 1.30 & $(0.56-3.07)$ & 0.54 & $28 / 33$ & 0.91 & $(0.53-1.57)$ & 0.93 & 0.90 & $(0.51-1.58)$ & 0.72 \\
\hline \multirow[t]{3}{*}{ Education } & High school or less & $113 / 80$ & Ref. & & & Ref. & & & $144 / 115$ & Ref. & & & Ref. & & \\
\hline & Two-year college & $74 / 54$ & 0.99 & $(0.62-1.57)$ & 0.96 & 1.02 & $(0.58-1.79)$ & 0.94 & $66 / 90$ & 0.60 & $(0.40-0.91)$ & 0.01 & 0.63 & $(0.42-0.96)$ & 0.03 \\
\hline & University & $27 / 45$ & 0.43 & $(0.24-0.76)$ & $<0.01$ & 0.33 & $(0.16-0.67)$ & 0.00 & $36 / 74$ & 0.40 & $(0.25-0.64)$ & $<0.01$ & 0.45 & $(0.28-0.73)$ & $<0.01$ \\
\hline
\end{tabular}

${ }^{\mathrm{a} O R}$ is adjusted for age.

${ }^{b}$ Multivariate adjusted for smoking state, leisure-time exercise, party, age of first children, breastfeeding and education. ${ }^{c}$ Multivariate adjusted for BMI, smoking state, and education. ${ }^{\mathrm{d}}$ Intensity of physical activity and education. Significant dates are showed in boldface. OR, odds ratio; Cl, confidence interval; BMI, body mass index. 
located $200 \mathrm{~kb}$ upstream of ESR1. The risk allele frequency of rs 3757318 is $6.6 \%$ in Europeans (HapMapCEU), 33\% in Chinese (HapMap-HCB) and 25\% in Japanese (HapMap-JTP) [19]. We found a 22\% risk allele frequency, consistent with HapMap-JTP. Thus, the risk allele frequency for rs3757318 varies between Europeans and Asians. In an analysis of the association between rs2046210 and rs12662670 as a surrogate for rs3757318 and breast cancer risk, Heins et al. found that that per allele OR for rs3757318 was higher in Asians (1.29, 95\% CI 1.19-1.41) than in Europeans (1.12, 95\% CI 1.081.17) [31]. These results suggest that screening for the rs3757318 genotype may be important in Asian women.

We also found that SNPs associated with breast cancer differed with regard to menses state, with rs2046210 and rs3803662 associated with breast cancer risk in premenopausal women. rs3803662 lies $8 \mathrm{~kb}$ upstream of TNRC9 and was found to have a significant association with breast cancer risk by Easton et al. [12]. TNRC9 is located on chromosome $16 \mathrm{q} 12$ and consists of seven exons. The protein encoded by this gene is a member of the high mobility group box (HMG-box) family. TNRC9 is expressed in brain and breast tissue, and has a higher expression level in breast cancer compared to that in normal tissue [37]. The risk allele frequency of rs3803662 is $24 \%$ in Europeans (HapMap-CEU), 72\% in Chinese (HapMap-HCB) and 60\% in Japanese (HapMap-JTP) [19]. Thus, Asian populations have a higher risk allele frequency than Europeans. However, Chen et al. found that rs3803662 was significantly associated with breast cancer in Europeans [17], but that this relationship was unclear in Asians [38]. Among the breast cancer-associated SNPs found in the current study, rs2046210 and rs3757318 are located near ESR1 and are related to breast cancer risk in Asians. To examine whether lifestyle factors associated with breast cancer risk vary in risk allele and non-risk allele carriers, we performed a subgroup analysis. Leisure-time exercise were associated with a decreased breast cancer risk in rs2046210 risk allele carriers. Although low-penetrance susceptibility SNPs may confer only a small effect on breast cancer risk alone, the risk for development of breast cancer in a risk allele carrier is about 1.2-1.3 fold higher than that in non-carriers. However, our results suggest that risk allele carriers can reduce their breast cancer risk through exercise, whereas obesity and smoking may increase breast cancer risk in non risk-allele carriers. An understanding of the mechanisms underlying the different lifestyle factors associated with breast cancer in rs2046210 and rs3757318 risk allele and non-risk allele carriers may clarify the effects of these SNPs located near ESR1. Examination of interactions between SNPs and lifestyle factors in a larger Japanese population is needed to confirm the current findings for SNPs, lifestyle factors and breast cancer.

\section{Conclusions}

This case-control study showed that rs2046210 and rs3757318 located near the ESR1 gene and rs3808662 located on TNRC 9 are associated with breast cancer risk in Japanese women. Our results suggest that leisure-time exercise can reduce the breast cancer risk in rs2046210 risk allele carriers, whereas smoking and obesity may increase the breast cancer risk in non-risk allele carriers. Further studies are required to confirm the validity of the association of these SNPs and lifestyle factors with breast cancer risk in the Japanese population.

\section{Abbreviations}

SNPs: Single nucleotide polymorphisms; WCRF/AICR: World Cancer Research Fund/American Institute for Cancer Research; NIC: National Cancer Institute; GWAS: Genome-wide association studies; LD: Linkage disequilibrium; BMI: Body mass index; MET: Metabolic equivalent; OR: Odds ratio;

Cl: Confidence interval; ERa: estrogen receptor a.

\section{Competing interests}

The authors declare that they have no competing interests.

\section{Authors' contributions}

NT designed the study. TM carried out genotyping, performed statistical analysis, and wrote the manuscript with NT. KN participated in genotyping and statistical analysis. TN, TI, TM, TS, JM, HD, SI, HK, KK, YI and YO obtained informed consent from subjects, collected blood samples and data from subjects, and provided advice on the study. YK designed the study and served as an advisor. All authors read and approved the final manuscript.

\section{Acknowledgements}

This study was supported by a Grant-in-Aid for Scientific Research (C) from the Ministry of Education, Culture, Sports, Science and Technology of Japan.

\section{Author details}

'Department of General Thoracic Surgery and Breast and Endocrinological Surgery, Okayama University Graduate School of Medicine, Dentistry, and Pharmaceutical Sciences, 2-5-1 Shikata-cho, Okayama-city, Okayama 700-8558, Japan. ${ }^{2}$ Department of Radiology, Okayama Saiseikai General Hospital, 1-17-18 Ifuku-cho, Okayama-city, Okayama 700-8511, Japan. ${ }^{3}$ Department of Breast Surgery, Okayama Rousai Hospital, 1-17-18 Chikkoumidorimachi, Okayama-city, Okayama 702-8055, Japan. ${ }^{4}$ Department of Breast Surgery, Kagawa Prefectural Cancer Detection Center, 587-1 Tougou-cho, Takamatu-city, Kagawa 761-8031, Japan. ${ }^{5}$ Department of Breast Surgery, Mizushima Kyodo Hospital, 1-1 Mizushima, Minamikasuga-cho Kurashiki-city, Okayama 712-8567, Japan. ${ }^{6}$ Department of Breast and Endocrinological Surgery, 5-4-16, Ban-cho, Takamatu-city, Kagawa 760-8557, Japan. ${ }^{7}$ Faculty of Medicine, Kinki University Hospital, 377-2 Ohnohigashi, Osakahayama-city, Osaka 589-8511, Japan.

Received: 26 July 2013 Accepted: 18 November 2013

Published: 1 December 2013

\section{References}

1. Matsuda A, Matsuda T, Shibata A, Katanoda K, Sobue T, Nishimoto H, The Japan Cancer Surveillance Research Group: Cancer incidence and incidence rates in Japan in 2007: a study of 21 population-based cancer registries for the Monitoring of Cancer Incidence in Japan (MCIJ) project. Jpn J Clin Oncol 2013, 43:328-336.

2. Ministry of Health, Ministry of Health, Labour and Welfare: Vital Statistics; 2011. http://www.mhlw.go.jp/toukei/saikin/hw/jinkou/geppo/nengai11/ kekka03.html\#k3_2.

3. World Cancer Research Fund/American Institute for Cancer Research: Food, Nutrition, Physical Activity, and the Prevention of Cancer: a Global Perspective. Washington DC: AICR; 2007

4. Gail MH, Brinton LA, Byar DP, Corle DK, Green SB, Shairer C, Mulvihill JJ: Projecting individualized probabilities of developing breast cancer for 
white females who are being examined annually. J Natl Cancer Inst 1989, 81:1879-1886.

5. Breast Cancer Risk Assessment Tool. http://www.cancer.gov/bcrisktool/ Default.aspx.

6. The Japanese Breast Cancer Society: Clinical Practice Guidelines for Breast Cancer. Tokyo: KANEHARA \& Co., Ltd.; 2012.

7. Hindorff LA, Sethupathy P, Junkins HA, Ramos EM, Mehta JP, Collins FS, Manolio TA: Potential etiologic and functional implications of genome-wide association loci for human diseases and traits. Proc Natl Acad Sci USA 2009, 106:9362-9367.

8. Resource Council, Science and Technology Agency \& the Government of Japan: Standard Tables of Food Composition in Japan. 5th edition. Tokyo: National Printing Bureau; 2005.

9. Ainsworth BE, Haskell WL, Whitt MC, Irwin ML, Swartz AM, Strath SJ, O'Brien WL, Bassett DR Jr, Schmitz KH, Emplaincourt PO, Jacobs DR Jr, Leon AS: Compendium of physical activities: an update of activity codes and MET intensities. Med Sci Sports Exerc 2000, 32(Suppl 9):498-504

10. Travis RC, Reeves GK, Green J, Bull D, Tipper SJ, Baker K, Beral V, Peto R, Bell J, Zelenika D, Lathrop M, Million Women Study Collaborators: Gene-environment interactions in 7610 women with breast cancer: prospective evidence from the Million Women Study. Lancet 2010, 375:2143-2151.

11. Turnbull C, Ahmed S, Morrison J, Pernet D, Renwick A, Maranian M, Seal S, Ghoussaini M, Hines S, Healey CS, Hughes D, Warren-Perry M, Tapper W, Eccles D, Evans DG, Breast Cancer Susceptibility, Collaboration, Hooning M, Schutte M, van den Ouweland A, Houlston R, Ross G, Langford C, Pharoah PD, Stratton MR, Dunning AM, Rahman N, Easton DF: Genome-wide association study identifies five new breast cancer susceptibility loci. Nat Genet 2010, 42:504-507.

12. Easton DF, Pooley KA, Dunning AM, Pharoah PD, Thompson D, Ballinger DG Struewing JP, Morrison J, Field H, Luben R, Wareham N, Ahmed S, Healey CS, Bowman R, Meyer KB, Haiman CA, Kolonel LK, Henderson BE, Le Marchand L, Brennan P, Sangrajrang S, Gaborieau V, Odefrey F, Shen CY, Wu PE, Wang HC, Eccles D, Evans DG, Peto J, et al: Genome-wide association study identifies novel breast cancer susceptibility loci. Nature 2007, 447:1087-1093.

13. Long J, Shu XO, Cai Q, Gao YT, Zheng Y, Li G, Li C, Gu K, Wen W, Xiang YB, Lu W, Zheng W: Evaluation of breast cancer susceptibility loci in Chinese women. Cancer Epidemiol Biomarkers Prev 2010, 19:2357-2365.

14. Qian B, Zheng $H, Y u$ H, Chen K: Genotypes and phenotypes of IGF-I and IGFBP-3 in breast tumors among Chinese women. Breast Cancer Res Treat 2011, 130:217-226.

15. Zheng W, Long J, Gao YT, Li C, Zheng Y, Xiang YB, Wen W, Levy S, Deming SL, Haines JL, Gu K, Fair AM, Cai Q, Lu W, Shu XO: Genome-wide association study identifies a new breast cancer susceptibility locus at $6 q 25.1$. Nat Genet 2009, 41:324-328

16. Stacey SN, Sulem P, Zanon C, Gudjonsson SA, Thorleifsson G, Helgason A, Jonasdottir A, Besenbacher S, Kostic JP, Fackenthal JD, Huo D, Adebamowo C, Ogundiran T, Olson JE, Fredericksen ZS, Wang X, Look MP, Sieuwerts AM, Martens JW, Pajares I, Garcia-Prats MD, Ramon-Cajal JM, de Juan A, Panadero A, Ortega E, Aben KK, Vermeulen SH, Asadzadeh F, van Engelenburg KC, Margolin S, et al: Ancestry-shift refinement mapping of the C6orf97-ESR1 breast cancer susceptibility locus. PLOS Genet 2010, 6:e1001029.

17. Chen MB, Wu XY, Shen W, Wei MX, Li C, Cai B, Tao GQ, Lu PH: Association between polymorphisms of trinucleotide repeat containing 9 gene and breast cancer risk: evidence from 62,005 subjects. Breast Cancer Res Treat 2011, 126:177-183

18. Hirakawa M, Tanaka T, Hashimoto Y, Kuroda M, Takagi T, Nakamura Y: JSNP: a database of common gene variations in the Japanese population. Nucleic Acids Res 2002, 30:158-162.

19. International HapMap Project. http://hapmap.ncbi.nlm.nih.gov.

20. McPherson $K$, Steel CM, Dixon JM: ABC of breast diseases. Breast cancerepidemiology, risk factors, and genetics. BMJ 2000, 321:624-628.

21. Nagata $\mathrm{C}, \mathrm{Hu} \mathrm{YH}$, Shimizu H: Effects of menstrual and reproductive factors on the risk of breast cancer: meta-analysis of case-control studies in Japan. Jpn J Cancer Res 1995, 86:910-915.

22. Yang XR, Chang-Claude J, Goode EL, Couch FJ, Nevanlinna H, Milne RL, Gaudet M, Schmidt MK, Broeks A, Cox A, Fasching PA, Hein R, Spurdle AB, Blows F, Driver K, Flesch-Janys D, Heinz J, Sinn P, Vrieling A, Heikkinen T, Aittomaki K, Heikkila P, Blomqvist C, Lissowska J, Peplonska B, Chanock S, Figueroa J, Brinton L, Hall P, Czene K, et al: Associations of breast cancer risk factors with tumor subtypes: a pooled analysis from the Breast Cancer Association Consortium studies. J Natl Cancer Inst 2011, 103:250-263.

23. Ma H, Bernstein L, Pike MC, Ursin G: Reproductive factors and breast cancer risk according to joint estrogen and progesterone receptor status: a meta-analysis of epidemiological studies. Breast Cancer Res 2006, 8:R43.

24. Gaudet MM, Gapstur SM, Sun J, Diver WR, Hannan LM, Thun MJ: Active smoking and breast cancer risk: original cohort data and meta-analysis. J Natl Cancer Inst 2013, 105:515-525.

25. Nagata C, Mizoue T, Tanaka K, Tsuji I, Wakai K, Inoue M, Tsugane S, Research Group for the Development and Evaluation of Cancer Prevention Strategies in Japan: Tobacco smoking and breast cancer risk: an evaluation based on a systematic review of epidemiological evidence in the Japanese population. Jpn J Clin Oncol 2006, 36:387-394

26. Kawai M, Kakugawa Y, Nishino Y, Hamanaka Y, Ohuchi N, Minami Y: Anthropometric factors, physical activity, and breast cancer risk in relation to hormone receptor and menopausal status in Japanese women: a case-control study. Cancer Causes Control 2013, 24:1033-1044.

27. Expert Consultation WHO: Appropriate body-mass index for Asian populations and its implications for policy and intervention strategies. Lancet 2004, 363:157-163.

28. Suzuki S, Kojima M, Tokudome S, Mori M, Sakauchi F, Wakai K, Fujino Y, Lin Y, Kikuchi S, Tamakoshi K, Tamakoshi A: Obesity/weight gain and breast cancer risk: findings from the Japan collaborative cohort study for the evaluation of cancer risk. J Epidemio/ 2013, 23:139-145.

29. Hussain SK, Altieri A, Sundquist J, Hemminki K: Influence of education level on breast cancer risk and survival in Sweden between 1990 and 2004. Int J Cancer 2008, 122:165-169.

30. Hajian-Tilaki K, Kaveh-Ahangar T, Hajian-Tilaki E: Is educational level asso ciated with breast cancer risk in Iranian women? Breast Cancer 2012, 19:64-70.

31. Ministry of Health, Labour and Welfare: Summary of Comprehensive Survey of Living Conditions; 2010. http://www.mhlw.go.jp/toukei/saikin/hw/k-tyosa/ktyosa10/3-6.html.

32. Kos M, Reid G, Denger S, Gannon F: Minireview: genomic organization of the human ERalpha gene promoter region. Mol Endocrinol 2001, 15:2057-2063.

33. Dahlman-Wright $K$, Cavailles V, Fuqua SA, Jordan VC, Katzenellenbogen JA, Korach KS, Maggi A, Muramatsu M, Parker MG, Gustafsson JA: International Union of Pharmacology. LXIV. Estrogen receptors. Pharmacol Rev 2006, 58:773-781.

34. Hein R, Maranian M, Hopper JL, Kapuscinski MK, Southey MC, Park DJ, Schmidt MK, Broeks A, Hogervorst FB, Bueno-de-Mesquit HB, Muir KR, Lophatananon A, Rattanamongkongul S, Puttawibul P, Fasching PA, Hein A, Ekici AB, Beckmann MW, Fletcher O, Johnson N, dos Santos SI, Peto J, Sawyer E, Tomlinson I, Kerin M, Miller N, Marmee F, Schneeweiss A, Sohn C, Burwinkel B, et al: Comparison of $6 q 25$ breast cancer hits from Asian and European Genome Wide Association Studies in the Breast Cancer Association Consortium (BCAC). PLoS One 2012, 7:e42380.

35. Cai Q, Wen W, Qu S, Li G, Egan KM, Chen K, Deming SL, Shen H, Shen CY, Gammon MD, Blot WJ, Matsuo K, Haiman CA, Khoo US, Iwasaki M, Santella RM, Zhang L, Fair AM, Hu Z, Wu PE, Signorello LB, Titus-Ernstoff L, Tajima K, Henderson BE, Chan KY, Kasuga Y, Newcomb PA, Zheng H, Cui Y, Wang F, et al: Replication and functional genomic analyses of the breast cancer susceptibility locus at $6 q 25.1$ generalize its importance in women of Chinese, Japanese, and European ancestry. Cancer Res 2011, 71:1344-1355.

36. Guo H, Ming J, Liu C, Li Z, Zhang N, Cheng H, Wang W, Shi W, Shen N, Zhao Q, Li D, Yi P, Wang L, Wang R, Xin Y, Zhao X, Nie X, Huang T: A common polymorphism near the ESR1 gene is associated with risk of breast cancer: evidence from a case-control study and a meta-analysis. PLoS One 2012, 7:e52445.

37. Jones JO, Chin SF, Wong-Taylor LA, Leaford D, Ponder BA, Caldas C, Maia AT: TOX3 Mutations in breast cancer. PloS one 2013, 8:e74102.

38. Liang J, Chen P, Hu Z, Shen H, Wang F, Chen L, Li M, Tang J, Wang H, Shen H: Genetic variants in trinucleotide repeat-containing 9 (TNRC9) are associated with risk of estrogen receptor positive breast cancer in a Chinese population. Breast Cancer Res Treat 2010, 124:237-241.

doi:10.1186/1471-2407-13-565

Cite this article as: Mizoo et al.: Effects of lifestyle and single nucleotide polymorphisms on breast cancer risk: a case-control study in Japanese women. BMC Cancer 2013 13:565. 